\title{
The Compromise Game: Two-Sided Adverse Selection in the Laboratory $\boxplus$
}

\author{
By Juan D. Carrillo and Thomas R. Palfrey*
}

\begin{abstract}
We analyze a game of two-sided private information where players have privately known "strengths" and can decide to fight or compromise. If either chooses to fight, the stronger player receives a high payoff and the weaker player receives a low payoff. If both choose to compromise, each player receives an intermediate payoff. The only equilibrium is for players to always fight. In our experiment, we observe frequent compromise, more fighting the lower the compromise payoff and less fighting by first than second movers. We explore several theories of cognitive limitations in an attempt to understand these anomalous findings. (JEL C91, D82)
\end{abstract}

A

major insight from theoretical research in information economics is that profitable agreements may be severely impeded by private information, and can even dry up completely. This was nicely illustrated in the famous market for lemons example (George A. Akerlof 1970) and studied in further detail by Roger B. Myerson and Mark Satterthwaite (1983) in a context of optimal contracting with two-sided private information. More generally, no-trade theorems show that rational, expected utility maximizing, Bayesian economic agents will not trade with each other on the basis of private information alone (Paul Milgrom and Nancy Stokey 1982, Stephen Morris 1994).

In this paper, we study the other side of the coin, namely a situation where exchanges (or other types of agreements) are not mutually beneficial and ask the following question: can private information induce agents to reach agreements that one of them will (ex post) regret? An all-too-familiar example, war, illustrates the problem. Suppose there are two nations, either of which would be better off conquering the other nation rather than coexisting peacefully, and would be worse off being conquered. If there is a war, the stronger country conquers the weaker one. Each

\footnotetext{
* Carrillo: Department of Economics, University of Southern California, Los Angeles, CA 90089-0253 and CEPR (e-mail: juandc@usc.edu). Palfrey: Division of the Humanities and Social Sciences, California Institute of Technology, Pasadena, CA 91125 (e-mail: trp@hss.caltech.edu). Part of this research was conducted while the first author was visiting Caltech. The hospitality of the hosting institution is greatly appreciated. We also gratefully acknowledge the financial support of the National Science Foundation (SES-0079301, SES-0450712, SES0094800), The Princeton Laboratory for Experimental Social Science, and The Princeton Center for Economic Policy Studies. We thank seminar audiences at Collège de France, Princeton University, Universidad Carlos III, Universitat Autonoma de Barcelona, the University of Pennsylvania, the Fundación Ramon Areces Conference on Experimental and Behavioral Economics in December 2006, and the 2006 ESA meeting in Tucson for comments, and Shivani Nayyar, Stephanie Wang, and Rumen Zarev for research assistance.

$\dagger$ To comment on this article in the online discussion forum visit the articles page at: http://www.aeaweb.org/articles.php?doi=10.1257/mic.1.1.151
} 
nation makes the choice to attack or not. They remain in peaceful coexistence if both choose not to attack, a war ensues otherwise. If one formalizes this problem, it is obvious that the strongest nation always has an incentive to attack the weakest one. Thus, a war is inevitable. More interestingly, the equilibrium is also war if the leader of each nation knows its own military strength but knows only the probability distribution of the other nation's strength (and therefore is uncertain over his chances of winning). This would be true, for example, even if the benefits of winning the war were only slightly greater than the benefits of peace; the costs of losing the war were enormous; and the uncertainty about the other nation's strength was large. The logic is much like the unraveling argument in adverse selection games. In deciding whether to attack or not, optimal decision making requires the agents to condition on their opponent choosing not to attack. Because weaker opponents are the ones who do not attack, this conditioning will lead stronger opponents to attack. Therefore, there will be a marginal strength level which is indifferent between peace and war. But this calculus will lead the opponent's marginal nonattackers to attack, and so forth. The only equilibrium is for the marginal strength type to be the weakest type. As developed in Section IA, the same logic applies to other situations where parties with conflicting goals and private information can reach an agreement that cannot ex post benefit both parties such as litigation, electoral debates, and firm competition.

Here, we report an experiment that investigates behavior in several variations of this two-sided asymmetric information environment. In all the variations, the equilibrium outcome predicted by the theory is the same-fighting ensues with probability one. We obtain three main results that are all inconsistent with standard game theory. First and foremost, fighting occurs much less often than predicted by theory. Rather than 1.0, we observe fight probabilities in the range of $0.50-0.70$. The outcome characterized by both agents compromising arises with surprising frequency, nearly one-quarter of the time in some sessions. In terms of our example, it means that contrary to the predictions of game theory, a war can be avoided if the military strengths of countries are privately (rather than publicly) known. Second, fight rates are affected by the compromise payoff. In both the sequential and simultaneous treatments, agents are less likely to fight the higher the compromise payoff. Third, in the sequential version, the strategies of first and second movers are different in two ways: second movers are more likely to fight than first movers; and the behavior of second movers is more responsive to strength and less erratic than that of first movers.

We also obtain some findings about individual behavior. Individual choice is consistent with the use of cutpoint strategies-fight if and only if strength is above a certain critical threshold. However, instead of the cutpoints being at (or at least close to) the minimum strength, as predicted by the theory, we find that players use cutpoints in an intermediate range. The use of cutpoint strategies indicates that subjects have some understanding of the game, and the source of violations of equilibrium has to do with the cognitive difficulty in choosing the cutpoint optimally. Perhaps more interesting, we find substantial heterogeneity in the choice of cutpoints across subjects, and also important differences in the distribution of cutpoints across treatments. Finally, all the results are robust with respect to experience, that is, there is only a limited evidence of learning. 
We then explore three recent theories of cognitive limitations in games, and analyze the data to investigate the extent to which the insights from these alternative theories can account for these anomalies. The three approaches we explore are equilibrium stochastic choice, levels of strategic sophistication, and naïve belief formation. The specification of our models for these three approaches are: the logit specification of Quantal Response Equilibrium theory (QRE); the Poisson specification of the Cognitive Hierarchy model $(\mathrm{CH})$; and a stochastic choice version of Cursed Equilibrium (CE), respectively. The estimated parameters for each model are relatively constant across treatments. There are some important differences in the predictions that lead to differences in the fit of the models, however. Only the QRE model captures the tendency of second movers to fight more often than first movers. The $\mathrm{CH}$ and $\mathrm{CE}$ models capture the aggregate tendency of players to fight with probability close to one when their strength is sufficiently high and with probability close to zero when their strength is sufficiently low. The $\mathrm{CH}$ model predicts that the distribution of individual cutpoints will be multimodal, clustered around three or four numbers, which is not reflected in the data. Finally, the best fit is obtained with a hybrid of QRE and CE, which combines cursedness and stochastic choice.

\section{The Theoretical Model}

We analyze the incentives of agents to compromise when they have conflicting objectives and asymmetric information. To this end, we study a class of games that have unique Nash equilibrium outcomes in which a compromise is never reached.

\section{A. Some Introductory Examples}

Consider two agents who must decide whether to split a surplus in a prespecified manner (compromise) or try to reap all the benefits (no compromise). Both agents have private but imperfect information about their likelihood of obtaining the benefits if they do not compromise and, possibly, also their value. The ex post sum of utilities may be higher or lower under compromise than under no compromise.

A myriad of situations fit this general description in addition to the example of international conflict described in the introduction. In a litigation, the defendant may offer a settlement to the plaintiff that can be accepted or not. Both parties have private knowledge of the strength of their case and the bias of the jury. In an electoral campaign, each candidate can drive his rival into a public debate, where some qualities of the contenders are revealed to voters, affecting the electoral outcome. In the absence of a debate, voters must rely on expected qualities. In a product market competition, firms offering horizontally differentiated products may start a research and deveopment $(\mathrm{R} \& \mathrm{D})$ race. The winner monopolizes the market and the probability of winning is proportional to the privately known quality of the firms research department. Alternatively, firms can avoid the race and share the market. In all these cases, there are two possible outcomes: settlement, peace, no debate, market sharing versus trial, war, debate, $R \& D$ race, respectively. The first outcome needs the agreement of both players, whereas each player can unilaterally force the second outcome. Payoffs depend on the state of the world, which is not realized or revealed until after 
all players have acted. Also, the utility of agents under the different outcomes may depend on some exogenously given private information parameters. Payoffs under agreement are typically determined by the status quo, whereas payoffs under no agreement are typically determined by a winner-takes-all rule. More generally, in the no agreement outcome, there is always one (ex post) winner and one (ex post) loser relative to the agreement outcome. The total surplus from compromise varies across these different applications, although we show below that the equilibrium does not depend on the compromise payoffs. Wars are typically costly and socially inefficient, so there is a peace dividend. Litigation also involves a waste of resources when compared to early settlements (but not compared to last minute settlements). Electoral debates are roughly neutral if candidates are only interested in winning the election. However, they provide information regarding the merits of different proposals, which can be valuable if candidates also care about policy outcomes. As for market competition, the profits of a monopolist are typically higher than the sum of profits of two duopolists, which suggests that firms' surplus increases when fighting for supremacy rather than splitting the market ${ }^{1}$

\section{B. Formalizing the Game}

We formalize the problem as follows. Denote by $s_{i} \in S_{i}$ and $s_{j} \in S_{j}$ the privately known "strength" of agents $i$ and $j$, with $i, j \in\{1,2\}$ and $i \neq j$ (case strength, military capacity, politician's talent, research quality). These values are drawn from continuous and commonly known distributions $F_{i}\left(s_{i} \mid s_{j}\right)$, possibly different and possibly correlated. For technical convenience, we assume strictly positive densities $f_{i}\left(s_{i} \mid s_{j}\right)$ for all $s_{i}$ and $s_{j}$. Agent $i$ chooses action $a_{i} \in A=\{\rho, \phi\}$, where $\rho$ stands for "retreat" and $\phi$ for "fight." If $a_{1}=a_{2}=\rho$, there is compromise (settlement, peace, no debate, no race) and the payoff of agent $i$ is $\beta_{i}\left(s_{1}, s_{2}\right)$. Otherwise, there is no compromise (trial, war, debate, race), and the payoff of agent $i$ is $\alpha_{i}\left(s_{1}, s_{2}\right)$ if $s_{i}>s_{j}$ and $\gamma_{i}\left(s_{1}, s_{2}\right)$ if $s_{i}$ $<s_{j}$, with $\alpha_{i}\left(s_{1}, s_{2}\right)>\beta_{i}\left(s_{1}, s_{2}\right)>\gamma_{i}\left(s_{1}, s_{2}\right)$ for all $i, s_{1}, s_{2}$. Note that, ex post, a compromise is always beneficial to one agent and detrimental to the other. The pair of strengths $\left(s_{i}, s_{j}\right)$ determines the winner and the loser. Payoffs under compromise and no compromise are given exogenously, although they may be unknown at the time of making the decision if they depend on $\left(s_{1}, s_{2}\right)$. Last, depending on the context, the socially efficient action may be compromise or no compromise, or it may even be a zero-sum game, $\alpha_{i}\left(s_{1}, s_{2}\right)+\gamma_{j}\left(s_{1}, s_{2}\right) \gtreqless \beta_{i}\left(s_{1}, s_{2}\right)+\beta_{j}\left(s_{1}, s_{2}\right)$ for all $s_{i}>s_{j}$.

\section{Equilibrium}

Given this structure, we can analyze the Perfect Bayesian Equilibrium (PBE) for the sequential version of the game, where agent 1 moves first and agent 2 moves second. We have the following result.

PROPOSITION 1: In all PBE of the game, the outcome is "no compromise."

\footnotetext{
${ }^{1}$ In these examples, we are not including the welfare of third parties such as society, voters, or consumers. These are also likely to be different across applications and outcomes.
} 


\section{PROOF:}

Suppose that there exists two sets, $\tilde{S}_{1} \subseteq S_{1}$ and $\tilde{S}_{2}\left(\tilde{S}_{1}\right) \subseteq S_{2}$, such that in a PBE of the game $a_{1}\left(s_{1}\right)=\rho$ and $a_{2}\left(s_{2}\right)=\rho$ with positive probability for all $s_{1} \in \tilde{S}_{1}$ and $s_{2} \in$ $\tilde{S}_{2}\left(\tilde{S}_{1}\right) \cdot 2$ Denote by $\bar{s}_{1}=\max _{s_{1} \in \tilde{S}_{1}}$ and $\bar{s}_{2}=\max _{s_{2} \in \tilde{S}_{2}\left(\tilde{S}_{1}\right)}$. According to this PBE, once agent 2 has observed $a_{1}=\rho$, the following inequality must be satisfied:

$$
\begin{gathered}
\int_{s_{1} \in \tilde{S}_{1}} \beta_{2}\left(s_{1}, s_{2}\right) d F_{1}\left(s_{1} \mid s_{1} \in \tilde{S_{1}}, s_{2}\right) \geq \int_{s_{1} \in \tilde{S}_{1} \cap s_{1}<s_{2}} \alpha_{2}\left(s_{1}, s_{2}\right) d F_{1}\left(s_{1} \mid s_{1} \in \tilde{S_{1}}, s_{2}\right) \\
\quad+\int_{s_{1} \in \tilde{S}_{1} \cap s_{1}>s_{2}} \gamma_{2}\left(s_{1}, s_{2}\right) d F_{1}\left(s_{1} \mid s_{1} \in \tilde{S_{1}}, s_{2}\right) \quad \forall s_{2} \in \tilde{S_{2}}\left(\tilde{S_{1}}\right),
\end{gathered}
$$

where the 1.h.s. is agent 2's expected payoff if $a_{2}=\rho$, and the r.h.s. is his expected payoff if $a_{2}=\phi$. This condition must hold in particular for $s_{2}=\bar{s}_{2}$. Since $\alpha_{2}\left(s_{1}, s_{2}\right)>$ $\beta_{2}\left(s_{1}, s_{2}\right)>\gamma_{2}\left(s_{1}, s_{2}\right)$, the inequality necessarily implies that $s_{1}>\bar{s}_{2}$ for some $s_{1} \in \tilde{S}_{1}$. Therefore, $\bar{s}_{2}<\bar{s}_{1}$. Now, agent 1's decision is relevant only if $a_{2}=\rho$. Thus, for the strategy described above to be a PBE, the following inequality must also hold:

$$
\begin{gathered}
\int_{s_{2} \in \tilde{S}_{2}\left(\tilde{S}_{1}\right)} \beta_{1}\left(s_{1}, s_{2}\right) d F_{2}\left(s_{2} \mid s_{1}\right) \geq \int_{s_{2} \in \tilde{S}_{2}\left(\tilde{S}_{1}\right) \cap s_{2}<s_{1}} \alpha_{1}\left(s_{1}, s_{2}\right) d F_{2}\left(s_{2} \mid s_{1}\right) \\
+\int_{s_{2} \in \tilde{S}_{2}\left(\tilde{S}_{1}\right) \cap s_{2}>s_{1}} \gamma_{1}\left(s_{1}, s_{2}\right) d F_{2}\left(s_{2} \mid s_{1}\right) \quad \forall s_{1} \in \tilde{S_{1}} .
\end{gathered}
$$

Using the same reasoning as before, $\bar{s}_{1}<\bar{s}_{2}$. Since both inequalities cannot be satisfied at the same time, $\tilde{S}_{1} \neq \emptyset$ and $\tilde{S}_{2}\left(\tilde{S}_{1}\right) \neq \emptyset$ cannot both occur in equilibrium.

The intuition is simple. In this class of games, agents know that good news for them is bad news for their rival. Thus, they have opposing interests for when to reach a compromise. As a result, whenever one agent wants to compromise, the other should not want to. For instance, country 1 has an incentive to stay in peaceful coexistence whenever its military strength, $s_{1}$, is low. However, this is precisely when country 2 wants to force a war. In other words, in these games, one agent's gain is always the other agent's loss (of same or different magnitude, it does not matter). Since a compromise is broken as soon as one agent does not find it profitable, the fact that an agent wants to deal implies that the other should not accept it, and vice versa. The bottom line is that, in equilibrium, compromises are never possible. We want to stress the generality of this result, which holds for any distribution of strengths (the same or different for both players) and any correlation between the players' strengths. Since the results hold for any payoffs satisfying $\alpha_{i}>\beta_{i}>\gamma_{i}$, it means that introducing risk aversion would not change the outcome of the game. Last, the

\footnotetext{
${ }^{2}$ Positive probability rather than probability 1 takes care of pure and mixed strategies at the same time.
} 
result is also unchanged if agents play simultaneously. Indeed, the only difference with the sequential game is that agent 2 will not compare his options conditional on having observed the choice of agent 1 . However, this does not make any difference since, both in the sequential and the simultaneous versions, each agent knows that his action is only relevant if the rival offers a compromise. Thus, the outcome of the Bayesian Nash Equilibrium (BNE) is always no compromise, just as it is for the PBE. This result is summarized as follows.

COROLLARY 1: The outcome of the game is still "no compromise" if agents are risk averse and/or if they announce their strategy simultaneously.

\section{Laboratory Experiment}

\section{A. Description of the Game}

This is a simplified version of the game described earlier. Each agent independently draws a number from a uniform distribution on $[0,1]$ and privately observes their own number, which we refer to as the player's strength, $s_{i}$. Agent 1 chooses whether to "fight," $\phi$, or "retreat," $\rho$. If 1 chooses $\phi$, then the game ends. The agent with highest strength receives a win payoff $H$, and the other agent receives a loss payoff $L(<H)$. If agent 1 chooses $\rho$, then it is agent 2's turn. If agent 2 chooses $\phi$, then as before, the agent with highest strength receives a payoff of $H$ and the other receives a payoff of $L$. If, instead, agent 2 also chooses $\rho$, then agent 1 and agent 2 each obtains a pre-specified "compromise payoff," $M$, where $L<M<H$. Thus, the main simplification relative to the theoretical model presented in Section I is that the win, lose, and compromise payoffs are all independent of $\left(s_{1}, s_{2}\right)$. Each player's strength only affects payoffs via the likelihood of winning under no compromise. We look at several variations on this game?

Variant 1. $H=1, L=0, M=0.50$ with sequential move.

Variant 2. $H=1, L=0, M=0.39$ with sequential move.

Variant 3. $H=1, L=0, M=0.50$ with simultaneous move.

Variant 4. $H=1, L=0, M=0.39$ with simultaneous move.

In our design, the total surplus under compromise is either smaller than $(M=0.39)$ or equal to $(M=0.50)$ the total surplus under no compromise. As discussed earlier, this specific choice of parameters fits some examples (firm competition, electoral debate) better than others (military conflict, litigation). There were at least two reasons for focusing on these values. First, the $M=0.50$ is a natural benchmark, corresponding to a constant sum game, where there is no difference in the efficiency

\footnotetext{
${ }^{3}$ The nominal payoffs in the experiment are: $H=95, L=5, M \in\{50,40\}$. Here, we present the scaled ver$\operatorname{sion}(x-5) / 90$.
} 
of the fight and compromise outcomes. Second, we wanted to choose another value of $M$ because the various behavioral theories we were testing all predict a negative comparative static effect of $M$ on the probability of fighting. Changes in either direction would allow us to test this. Our choice of a lower value of $M$ was made because we thought it was the more interesting direction, since, for $M<0.5$, always fighting is not only still the unique Nash equilibrium but is also ex ante efficient and ex ante fair. This allows us to better distinguish social preference or fairness-based explanations for excessive compromise (see Section IVE) from behavioral models based on cognitive limitations (see Sections IVA to IVD), and also gives Nash equilibrium its best shot. If the excessive compromise disappears, this would lend support for social preference theories based on ex ante fairness and efficiency, whereas, if the excessive compromise persists, it lends support for the models of cognitive limitations.

\section{B. Relation to the Experimental Literature}

Some related games have been studied in the laboratory. Below we describe two simultaneous games of multi-sided asymmetric information, two sequential games of one-sided asymmetric information and one static game of full information.

The Betting Game.-(Doron Sonsino, Ido Erev, and Sharon Gilat 2001; Ylva Sovic 2004; Colin F. Camerer, Palfrey, and Brian W. Rogers 2006). An asset yielding a fixed surplus can be traded between agents who have private information. Trade occurs only if both agents agree. As in our game, all the BNE imply no trade. There are three main differences. First, the risky outcome requires agreement in the betting game, whereas the safe outcome requires agreement in the compromise game. Second, the information structure is simpler than it is in ours. There are only four possible states, and in two of them one agent has full information. The common knowledge of this information partition triggers very naturally unraveling to no trade. This special partition is also likely to facilitate learning. Third, a sequential version of the betting game has not been studied.

Auction of a Common Value Good and the Winner's Curse.-(John H. Kagel and Dan Levin 2002). As in our game, agents will play suboptimally if they do not anticipate the information contained in the rival's action. Our game allows for some simple comparative statics (different timings and different compromise payoffs). Also, our BNE and PBE are simple to compute.

Adverse Selection Game.-(Akerlof 1970). This game also predicts some unraveling. However, the robust conclusion is the existence of a cutoff below which there is agreement or trade and above which there is not. This cutoff can be the lower bound (i.e., never agree as in our game), but it can also be the upper bound (i.e., always agree) or an interior value, depending on the parameters of the game. William Samuelson and Max Bazerman (1985) show that the probability that buyers engage in unfavorable trades is increasing in the complexity of the adverse selection game. Charles A. Holt and Roger Sherman (1994) prove that buyers may underbid or overbid depending on the treatment conditions. Note that because it is one-sided 
asymmetric information, the buyer's action has no signaling value. There have also been several market experiments with informed sellers and asymmetric information about product quality (Michael Lynch et al. 1984).

Blind Bidding Game.-(Robert Forsythe, R. Mark Isaac, and Palfrey 1989). This experiment determines whether an informed seller reveals the quality of his good to an uninformed buyer. Full revelation occurs because the seller with the highest quality good always has an incentive to announce it, then so does the seller with the second highest quality good, and so on. However, there is no role for the key effect of our game, namely the anticipation of information conveyed by the rival's action.

Beauty Contest.-(Rosemarie Nagel 1995). As in our game, best response dynamics predicts unraveling for a wide range of parameters. Since the beauty contest is a static game of complete information, the details of convergence are different. Even the most naïve learning rules (such as "play optimally given the outcome in the past round and assuming that nobody else revises his strategy") predict rapid convergence if the beauty contest game is played repeatedly. The experimental data confirms this prediction, and a natural question is whether a similar convergence pattern is found in the compromise game.

\section{Experimental Design and Procedures}

We conducted five sessions with a total of 56 subjects, using a simple $2 \times 2$ design. The subjects were registered Princeton University students who were recruited by e-mail solicitation, and all sessions were conducted at The Princeton Laboratory for Experimental Social Science. All interaction in a session was computerized using an extension of the open source software package Multistage Games ${ }^{4}$ No subject participated in more than one session. The two dimensions of treatment variation were the compromise payoff ( $M=0.50$ versus $M=0.39)$ and the order of moves (simultaneous versus sequential play). In each session, subjects made decisions during 40 rounds, with $M$ fixed throughout the session. Half of the subjects participated in sessions with $M=0.39$, and half of the subjects participated in sessions with $M$ $=0.50$. In all sessions, we set $H=1$ and $L=0$. Each subject played exactly one game with one opponent in each round, with random rematching after each round. At the beginning of each round, $t$, each subject was independently assigned a new strength, $s_{i t}$, drawn from a uniform distribution on $[0,1]{ }^{5}$ Each subject observed his own strength but had to make the fight-retreat decision before observing the strength of the subject they were matched with. The opponent's strength was revealed only at the end of the round.

At the beginning of each session, instructions were read by the experimenter, who was standing on a stage in the front of the experiment room and who fully

\footnotetext{
${ }^{4}$ Documentation and instructions for downloading the software can be found at http://multistage.ssel. caltech.edu.

${ }^{5}$ In the experimental implementation of payoffs, the $H$ and $L$ payoffs paid off $\$ 0.57$ and $\$ 0.03$, respectively. The compromise payoff, $M$, was scaled accordingly at $\$ 0.30$ and $\$ 0.24$ for the two treatments.
} 
explained the rules, information structure, and client GUI for the simultaneous move game. A sample copy of the instructions is in the Web Appendix (http://www.aeaweb. org/articles.php?doi=10.1257/mic.1.1.151). After the instructions were finished, two practice rounds were conducted, for which

\begin{tabular}{ccc}
\multicolumn{3}{c}{ Table 1-Session Details For the } \\
Experiment \\
\hline \hline Session & No. of subjects & $M$ \\
\hline 1 & 8 & 0.50 \\
2 & 8 & 0.50 \\
3 & 12 & 0.50 \\
4 & 14 & 0.39 \\
5 & 14 & 0.39 \\
\hline
\end{tabular}
subjects received no payment. After the practice rounds, there was an interactive, computerized comprehension quiz that all subjects had to answer correctly before proceeding to the paid rounds. For the first 20 paid rounds of a session, subjects played the simultaneous version of the game. At the end of round 20, there was a brief instruction period during which rules for the sequential version of the game were explained. In each match of the sequential version, one of the two players was selected randomly to be the first mover. After the first mover made a fight-retreat choice, the second mover was informed of that choice, but was not informed of the strength of the first mover. If the first mover's choice was fight, the second mover had no choice, and simply clicked a button on the screen labeled "continue." If the first mover's choice was retreat, the second mover had a choice between fight and retreat. After the second mover made a choice, the match ended and the strength levels and outcome were revealed. The subjects then participated in 20 additional rounds of the sequential version of the game, with opponents, roles (first or second mover), and strengths randomly reassigned at the beginning of each round. Subjects were paid the sum of their earnings over all rounds in cash, in private, immediately following the session. Sessions averaged one hour in length, and subject earnings averaged \$25. Table 1 displays the pertinent details of the five sessions.

\section{A Descriptive Analysis of the Results}

In this section, we provide a descriptive analysis of the experimental results. We discuss the main aggregate features of the data, including the mean rates of fight and retreat (overall and as a function of strength) and explore time trends. We compare the data to two natural benchmarks. The first benchmark is Nash equilibrium, in which all players always choose $\phi$ regardless of strength (and, in the sequential version, regardless of the choice of the first mover). A second, weaker benchmark is the type-independent model, where the probability of fighting is independent of strength. We study the differences in probabilities of fighting as a function of the compromise payoff and the timing of the game. Last, we analyze the data at an individual level. For each player, we estimate a decision rule that maps strength into the probability of fighting.

\section{A. Aggregate Fight Rates Unconditional on Strength}

The simplest cut at the data is to compare the relative frequencies of choosing fight versus retreat, without conditioning on the actual draws of $s_{i}$. Table 2 shows the 
TABle 2-Unconditional Fight Rates

\begin{tabular}{llccc}
\hline \hline Order & Position & $M=0.39$ & $M=0.50$ & Pooled \\
\hline Sequential & First & $0.589(280)$ & $0.538(264)$ & $0.564(544)$ \\
Sequential & Second & $0.643(115)$ & $0.566(122)$ & $0.603(237)$ \\
Sequential & Both & $0.605(395)$ & $0.547(386)$ & $0.576(781)$ \\
Simultaneous & - & $0.657(560)$ & $0.573(560)$ & $0.616(1120)$ \\
Pooled & & $0.636(955)$ & $0.562(946)$ & $0.599(1901)$ \\
\hline
\end{tabular}

relative fight rates in the experiment, broken down by compromise payoff and order of moves, with the number of observations in parentheses. 6

There are several differences in fight rates across treatments and conditions.

First, there is a clear difference between $\phi$ rates in the $M=0.39$ and the $M=0.50$ treatments. Fighting is chosen approximately 13 percent more frequently when the compromise dividend is lower, and this difference is statistically significant $(p<0.01){ }^{7}$ This difference is observed in both the sequential and simultaneous treatments, for both the first and second movers separately, and these differences are all approximately the same magnitude. In looking at subsamples based on treatment or condition, the lower significant levels reflect the relatively small number of observations in each treatment: $p=0.05$ level for the simultaneous treatment (most observations), $p=0.10$ level for the sequential treatment, and $p>0.10$ for first movers and second movers, separately.

Second, first movers in the sequential game fight less frequently than second movers, both in the 0.39 and the 0.50 treatments. The differences in means are not statistically significant.

Third, there is more fighting in the simultaneous treatment than in the sequential treatment. These results are mirrored in a simple probit dummy variable regression, where the dependent variable is whether a subject chose to fight and the independent variables are $M$, "sim" (= 1 for the simultaneous treatment), and "role" (= 1 for second movers). The estimated coefficients and standard errors are given in Table 3.

The logic of the game suggests that, over time, learning should lead to unraveling. That is, perceptive players should be able to realize that they will improve their payoff by adopting a cutoff strategy lower than the cutoff strategy used by their opponent. Given the symmetry of the game, they should realize that perceptive opponents will also notice this. The unravelling logic may be responsible for the higher fighting rates of second relative to first movers. It also suggests that $\phi$ rates should be increasing over time in all treatments. We investigate this hypothesis by breaking the data down into early and late matches. In each session, there were 20 rounds each of the sequential and the simultaneous games. We code the choices in

\footnotetext{
${ }^{6}$ Due to a software error, four rounds of data in the sequential $M=0.50$ treatment were mistakenly conducted as practice rounds. Hence, there are slightly less observations in this treatment than in the other treatments.

${ }^{7}$ The significance levels reported here and later in the paper are based on standard tests treating the observations as independent. Later in the paper, we consider individual effects and learning.
} 
Table 4-Unconditional Fight Rates by Experience LeVel

\begin{tabular}{|c|c|c|c|c|c|}
\hline \multirow[b]{2}{*}{ Order } & \multirow[b]{2}{*}{ Role } & \multicolumn{2}{|c|}{$M=0.39$} & \multicolumn{2}{|c|}{$M=0.50$} \\
\hline & & Inexperienced & Experienced & Inexperienced & Experienced \\
\hline Sequential & First & $0.564(140)$ & $0.614(140)$ & $0.484(124)$ & $0.586(140)$ \\
\hline Sequential & Second & $0.672(61)$ & $0.611(54)$ & $0.484(64)$ & $0.655(58)$ \\
\hline Simultaneous & - & $0.611(280)$ & $0.704(280)$ & $0.582(280)$ & $0.564(280)$ \\
\hline
\end{tabular}

the first ten rounds of each version of the game as "inexperienced" and the last ten rounds of each version as "experienced." Table 4 presents the $\phi$ rates broken down by experience level. The number of observations is in parenthesis.

The effect of experience on the unconditional $\phi$ rates is ambiguous. In four of the six comparisons, the $\phi$ rate increases, as hypothesized, although it remains well below 1 . All four such differences are statistically significant. In two of the six comparisons, $\phi$ decreases, but these two changes are not significant. Furthermore, the two treatments where $\phi$ decreases have no apparent relationship to each other (simultaneous with $M=0.50$ and the second player in sequential with $M=0.39$ ).

\section{B. Aggregate Fight Rates Conditional on Strength}

The analysis above, while providing a useful sketch of the results, falls short of giving a complete picture of the aggregate data because the unconditional $\phi$ rates are not a sufficient statistic for the actual strategies. A behavior strategy in each game is a probability of choosing $\phi$ conditional on $s$. By aggregating across all the (strength, action) paired observations for a treatment, we can graphically display the aggregate empirical behavior strategy, and then compare this strategy across treatments. Figure 1 shows six graphs. The graphs on the left correspond to $M=0.39$, and the graphs on the right are for $M=0.50$. The middle and bottom graphs are for the first and second movers in the sequential treatment, and the top graphs are for the simultaneous movers. The strength is on the horizontal axis on a scale of 0 to 100 , and the empirical fighting frequencies are on the vertical axis on a scale of 0 to 1 . Thus, for example, if all subjects were to choose the same cutoff strategy, $s^{*}$, then we would observe a step function, with a probability of fighting equal to zero below $s^{*}$ and equal to one above $s^{*}$. Note that functions need not be monotonically increasing, although we expect that players with higher strength will be more likely to fight. The empirical frequencies are aggregated into bins of five units of strength $(1-5,6-10$, etc.) along the horizontal axis, with $\phi$-probabilities in the vertical axis.

These graphs suggest that second movers in the sequential version of the game behave differently from first movers in at least two ways. Second movers fight more than first movers. If one looks at the point in the graph where the fight probabilities first reach 50 percent, this switchpoint is in the high 20s for second movers in both the 0.39 and 0.50 treatments, while it is in the mid- to high 30 s for simultaneous movers and even higher for the first movers in the sequential treatment. These results are also supported by a probit regression similar to the one reported in Table 3, but including the independent variable $s$ to control for strength. The coefficients and $t$-statistics are reported in Table 5. Two important new results emerge when 


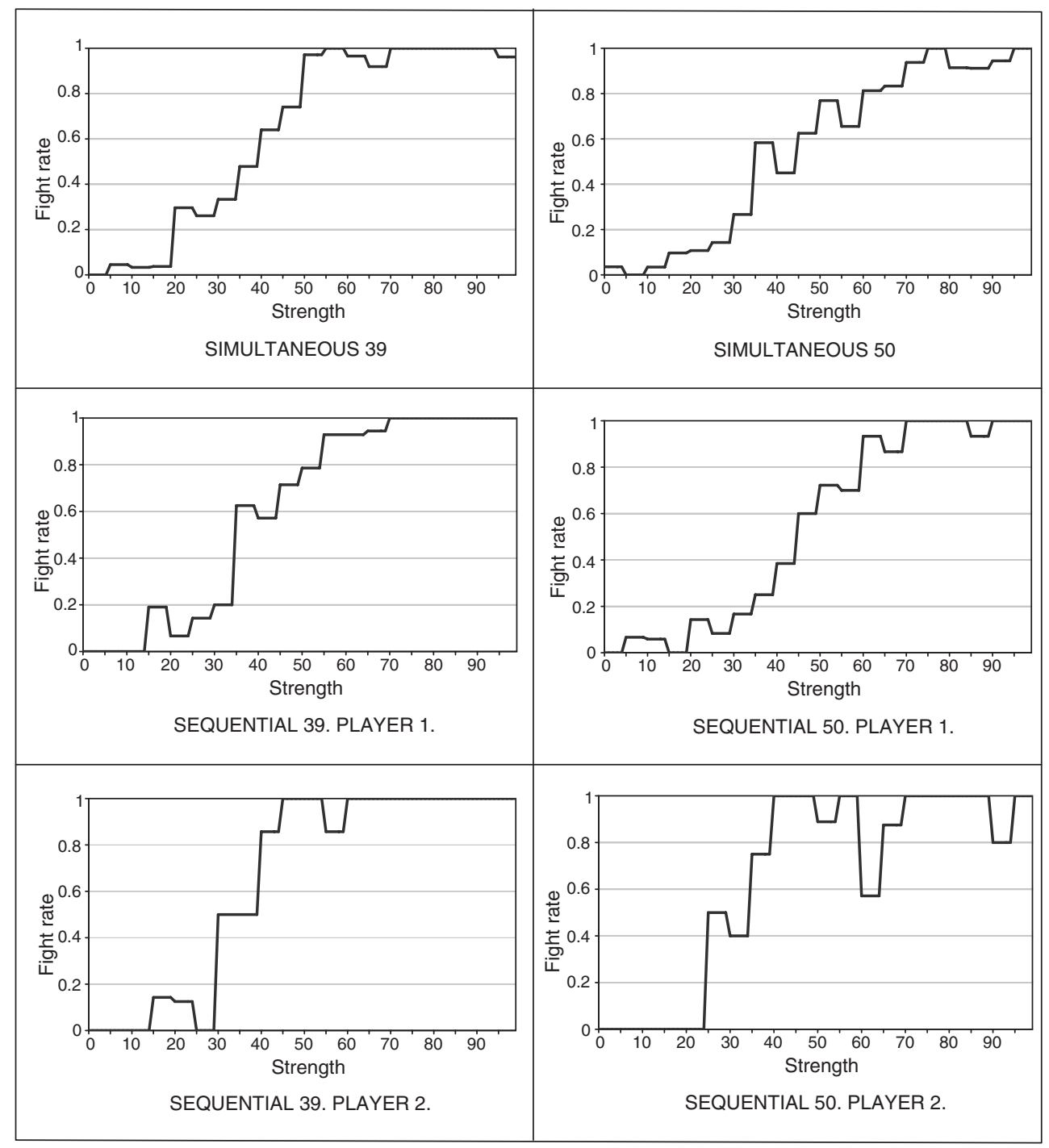

Figure 1. EMPIRICAL Fight RATES

Table 5-Probit Regression of Fight Rates, CONTROLling For Strength

\begin{tabular}{lccc}
\hline \hline & Coefficient & Standard error & $t$-statistic \\
\hline Constant & -0.487 & 0.376 & -1.296 \\
$M$ & -0.041 & 0.008 & -4.906 \\
sim & 0.076 & 0.093 & 0.818 \\
role & 0.229 & 0.139 & 1.640 \\
$s$ & 0.054 & 0.002 & 26.364 \\
\hline
\end{tabular}

controlling for strength. First, the difference in fight rates between the simultaneous and sequential treatments, which was significant without controlling for $s$, is no longer significant, indicating that the earlier finding was due to the different realizations of $s$ in the two samples. Second, the coefficient on "role" is now marginally significant, suggesting that the variation in sample draws of $s$ obscured the differences between the fight rates of first and second movers.

The figure also suggests that second movers display less erratic behavior, in the sense that for low values they (almost) never fight and for high values they (almost) 
Table 6-Probit Comparing Slope of Response for First and Second Movers

\begin{tabular}{lcccccc}
\hline \hline Position & $M$ & Constant & Slope & $-\ln L$ & $\%$ pred. & Observations \\
\hline First mover & 0.39 & $-2.73(0.307)$ & $0.068(0.0071)$ & 76.7 & 88.6 & 280 \\
Second mover & 0.39 & $-3.11(0.585)$ & $0.088(0.0016)$ & 21.6 & 92.2 & 115 \\
First mover & 0.50 & $-2.53(0.283)$ & $0.056(0.0057)$ & 83.4 & 87.1 & 264 \\
Second mover & 0.50 & $-1.83(0.162)$ & $0.044(0.0063)$ & 45.6 & 86.9 & 122 \\
\hline
\end{tabular}

Table 7-Cutpoint Summary Statistics

\begin{tabular}{lcccc}
\hline \hline Condition & $M$ & Median estimated cutpoint & Percentage misclassified & Empirical optimum \\
\hline Simultaneous & 0.39 & 37.5 & 3.4 & 17 \\
Simultaneous & 0.50 & 45.5 & 3.7 & 26 \\
First mover & 0.39 & 42.5 & 2.1 & 15 \\
First mover & 0.50 & 42.0 & 2.3 & 18 \\
Second mover & 0.39 & 36.0 & 0.0 & 18 \\
Second mover & 0.50 & 38.0 & 0.8 & 26 \\
\hline
\end{tabular}

always fight. This is reflected in a steeper response curve for player 2 shown in Figure 1. Table 6 describes aggregate behavior by treatment and position via a probit model, where the independent variables are strength and a constant term. The steeper response by the second mover is confirmed for the 0.39 treatment, where the slope of the second mover's response curve is significantly steeper than the slope of the first mover. There is no significant difference in the slope coefficient for the 0.50 treatment.

\section{Individual Cutpoint Analysis}

In order to address the question of conditional fight rates, treatment effects, and differences between first and second movers more carefully, we turn to an analysis of individual choice behavior. If subjects use cutpoint decision rules, how do cutpoints vary across treatments? How much variation is there across individuals? How consistent is individual behavior with cutpoint decision rules? We document that there is some heterogeneity across subjects but, more importantly, the distribution of these cutpoint strategies varies systematically across treatments.

In order to estimate cutpoint decision rules, we use a simple optimal classification procedure, similar to Palfrey and Jeffrey E. Prisbrey (1996) and Alessandra Casella, Andrew Gelman, and Palfrey (2006). For each subject and each condition the subject is in, we look at the set of strengths they were randomly assigned and the corresponding fight/retreat decision they made. For any hypothetical cutpoint strategy for an individual subject, we can then ask how many of these decisions are correctly classified. For example, if in some round a subject with strength 40 chose $\phi$, the decision would be classified correctly only if the hypothetical cutpoint were less than or equal to 40 . We then use the hypothetical cutpoint with the fewest misclassified decisions as the estimate for that individual and condition. If there are multiple best-fitting cutpoints, we take the average. Table 7 presents some cutpoint summary statistics.

Several observations are immediate. First, very few decisions are misclassified. In each of the simultaneous treatments, 16 of 28 subjects are perfectly classified. 
In the sequential conditions, the number of perfectly classified subjects range from 23 to 28 of 28 . The worst case of misclassification was one subject in the simultaneous, $M=0.50$ treatment who has 5 misclassified observations. Thus, with rare exceptions, subjects use cutpoint strategies. In our setting, it establishes that subjects have a basic understanding of the game, even if they do not play the Nash equilibrium strategy. Our subjects follow the first step of the logic (best responses are cutpoints) but fail at the second one (equilibrium unraveling). Although interesting, this result is not the central point of the section. We document it to justify our approach of comparing the distributions of estimated cutpoints across experimental conditions.

The key questions concern whether the distribution of cutpoints varies systematically across treatments and, in the sequential games, between first and second movers. We also want to understand whether these systematic variations are consistent with the descriptive findings based on aggregate data described in the previous section. Table 7 reports the median estimated cutpoint across all subjects, and the percentage of misclassified decisions by condition. The median cutpoints mirror the aggregate fight rates by treatment and condition, as reported in previous sections. Cutpoints are lower (more fighting) in $M=0.39$ than in $M=0.50$ treatments. They are lower for second movers than first movers for both the $M=0.39$ and $M=0.50$ treatments, and they are lower for second movers than for players in the simultaneous condition for both the $M=0.39$ and $M=0.50$ treatments. There is no systematic difference between first movers and players in the simultaneous condition. Last, second movers have fewer classification errors (therefore, steeper response curves) than first movers.

As further evidence, we consider how the entire distribution of individual estimated cutpoints varies across treatments. Figure 2 displays the estimated cumulative frequency distribution of individual cutpoints used by our subjects for all the treatments. They are broken down by position in the sequential treatment. The horizontal axis represents cutpoints ranging from 0 to 100. The vertical axis indicates how many of the subjects in each treatment or position (out of 28) were using a cutpoint less than or equal to that number.

These distributions exhibit a wide range of estimated cutpoints with few above 60 or below 20. In all treatments and conditions, there is heterogeneity that is both significant (one easily rejects the hypothesis that all subjects use the same cutpoint for any of these conditions) and substantial. The distributions are also different across treatments and conditions. Also noteworthy is that distributions are never concentrated around particular cutpoints (i.e., step distribution functions), but they are smoothly and uniformly increasing over the range.

Finally, one can compare the distribution of cutpoints used by players in the game to the cutpoint that would be optimal, given the actual frequencies of fighting in the experiment. These "empirically optimal" cutpoints are given in the last column of Table 7. The optimal cutpoints are generally about one-half times the corresponding median estimated cutpoints. Many, but not all, players are "fooled" by this game, in the sense that they set cutpoints that are too high. We find that 20 percent of the estimated cutpoints are within 5 units of strength of the optimal cutpoint, and these subjects are leaving essentially no money on the table. Of the 


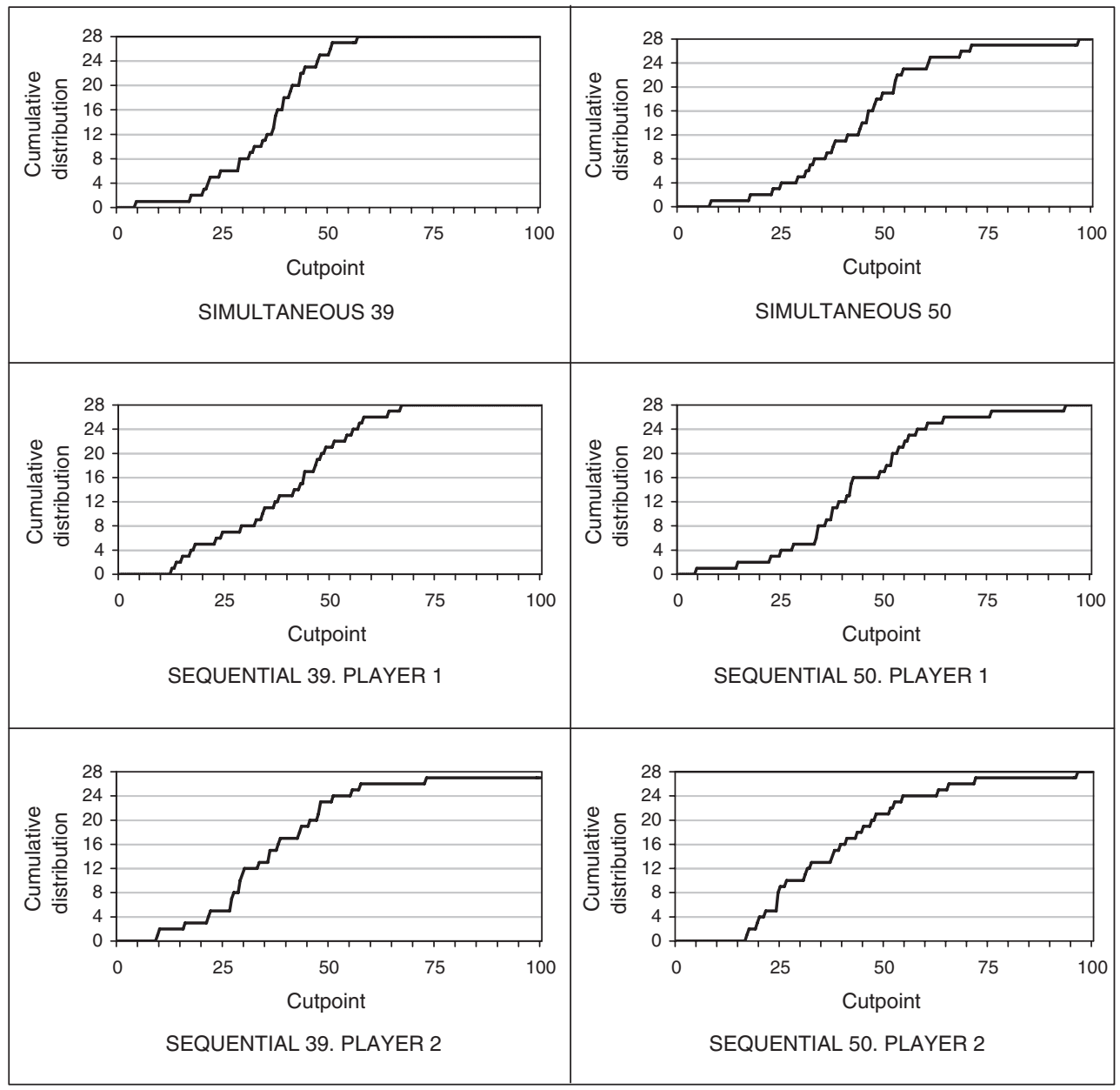

Figure 2. Distribution of Cutpoints by Condition

remaining estimated cutpoints, 7 percent are below the optimal cutpoint by at least 5 strength units, and 73 percent are above the optimal cutpoint by at least 5 units.

\section{Summary of Descriptive Analysis}

The main findings of our analysis, so far, can be summarized as follows:

- Unconditional fight probabilities range from about 0.5 to 0.7 , depending on the treatment and condition, falling far short of the theoretical prediction of 1.0.

- In all treatments, the probability of fighting conditional on strength increases monotonically from virtually 0 for strengths below 20 to virtually 1 for strengths above 60 if $M=0.39$ or above 70 if $M=0.50$.

- The compromise payoff, $M$, affects behavior, with less fighting when the compromise payoff is higher. 
- There are some differences between the sequential and simultaneous treatments. Most striking is that second movers behave differently than first movers. The former display more fighting and less erratic behavior (when $M=0.39$ ) than the latter. For a theory to explain this pattern, it must predict that observing the behavior of the rival before making inferences and choices leads to systematic differences compared with just conditioning on a hypothetical event.

- We do not find consistent evidence of learning. A possible explanation is the insufficient feedback provided to players (only the rival's strength and outcome is revealed at the end of each round). However, given that the order of moves matters, one would expect that second movers would use their experience in that role when they subsequently play as first mover.

- The vast majority of subjects use cutpoint strategies with very few deviations. Across all treatments, over 97 percent of individual behavior is consistent with cutpoint strategies. This shows their understanding, at least at an intuitive level, that the expected payoff differential between $\phi$ and $\rho$ increases with $s$, and possibly an even deeper understanding that the best reply in this game is always a cutpoint strategy. It also justifies the analysis that focuses on estimated cutpoints.

- The distribution of these cutpoints varies by condition in ways that mirror the differences in the aggregate fight rates. The empirical distribution of individual cutpoints is smooth.

\section{Alternative Behavioral Models}

Obviously, the Nash equilibrium model is inconsistent with our data. In this section, we consider several alternative models to explain the excessively low fight rates. Note that this game is easily solved by iterated dominance but only using weak rather than strict dominance. Denote a strategy as a function that maps strength into a probability of fighting $q:[0,1] \rightarrow[0,1]$. First, note that any strategy $q$ that assigns $q(1)<1$ is dominated weakly by the strategy $q^{\prime}$, where $q^{\prime}(s)=q(s)$ for all $s \neq 1$ and $q^{\prime}(1)=1$. In the experiments, the type distribution was discrete, so once we eliminate all those strategies, then any strategy $q$ that assigns $q(0.99)<1$ and $q(1)$ $=1$ is weakly dominated by the strategy $q^{\prime}$, where $q^{\prime}(s)=q(s)$ for all $s \neq 0.99$ and $q^{\prime}(0.99)=1$, and so forth 9 On the other hand, rationalizability does not eliminate any strategy, since every strategy is a weak best response to the equilibrium strategy, $q^{*}(s)=1$ for all $s$.

We consider two categories of models. Models in the first category have their foundations in cognitive limitations, and they all have features that admit the possibility of observing weakly dominated strategies. We study three models within this class: quantal response equilibrium or QRE (Richard D. McKelvey and Palfrey

\footnotetext{
${ }^{8}$ Recall that, in our design, all players gain experience as both first and second movers. That is, our data on first and second movers are all coming from the same subjects. Subjects apparently do not draw inferences from their own decision making in different roles about how other subjects behave in those roles.

${ }^{9}$ For the $M=0.50$ game, at the last iteration, a player with the lowest strength, $s=0.01$, is indifferent between $\phi$ and $\rho$, and therefore, there is an equilibrium with $s=0.01$ types choosing $\rho$ and all other types choosing $\phi$. For the $M=0.39$ game, the iteration continues all the way down, and the only equilibrium is $q^{*}(\mathrm{~s})=1$ for all $s$.
} 
1995), cognitive hierarchy or CH (Camerer, Teck-Hua Ho and Juin-Kuan Chong 2004), and cursed equilibrium or CE (Eric Eyster and Matthew Rabin 2005) 10 We also consider some variations that allow for heterogeneity or hybridization between models such as the truncated quantal response equilibrium or TQRE (Camerer, Palfrey, and Rogers 2006). This hybrid version allows us to understand better how different models capture different features of the observed behavior. Models in the second category have their foundations on the existence of systematic deviations from self-interest—social preferences, fairness motives, reciprocity, or altruism. For reasons we discuss later, we estimate only models in the first category.

\section{A. Quantal Response Equilibrium}

Quantal response equilibrium applies stochastic choice theory to strategic games. It is motivated by the idea that a decision maker may take a suboptimal action, and the probability of doing so is increasing in the expected payoff of the action. In a regular QRE (Jacob K. Goeree, Holt, and Palfrey 2005), one simply replaces the best response correspondence used to characterize Nash equilibrium with a quantal response function that is continuous and monotone in expected payoffs. That is, the probability of choosing a strategy is a continuous increasing function of the expected payoff of using that strategy, and strategies with higher payoffs are used with higher probability than strategies with lower payoffs. A quantal response equilibrium is then a fixed point of the quantal response mapping. In a logit equilibrium, for any two strategies, the log odds of the choice probabilities are proportional to the difference in expected payoffs, where the proportionality factor, $\lambda$, is a measure of responsiveness of choices to payoffs. That is,

$$
\ln \left[\frac{\sigma_{i j}}{\sigma_{i k}}\right]=\lambda\left[U_{i j}-U_{i k}\right]
$$

where $\sigma_{i j}$ is the probability that player $i$ chooses strategy $j$, and $U_{i j}$ is the corresponding expected payoff in equilibrium. Note that a higher $\lambda$ reflects a "more precise" response to the payoff differential. The polar cases $\lambda=0$ and $\lambda \rightarrow+\infty$ correspond to random choice and Nash equilibrium, respectively.

Specification of the QRE Model.-We consider two different specifications of the logit equilibrium version of QRE. The first specification takes an interim approach and analyzes the game in behavioral strategies. This approach corresponds to the agent QRE (AQRE) of McKelvey and Palfrey (1998). Conditional on player 1's strength and given the AQRE behavioral strategies used by player 2, the log-odds of player 1 choosing retreat versus fight is proportional to the difference in expected payoffs between retreat and fight, and similarly for player 2 .

The second analyzes the game in ex ante strategies, and assumes players choose stochastically over possible plans for whether or not to fight as a function of strength.

\footnotetext{
${ }^{10}$ Some preliminary findings about $\mathrm{CH}$ and CE are discussed in Stephanie Wang (2006) with permission of the authors.
} 
Because the set of all possible pure strategies in our game is huge $\left(2^{100}\right)$, we are forced to consider only a subset of such strategies. The natural restriction is to consider only monotone strategies, i.e., cutpoint strategies. This is a natural criterion since monotone strategies are always best responses and, furthermore, any nonmonotone strategy is dominated weakly by a monotone strategy. This also reduces the set of pure strategies to a small enough number (100) that estimation is possible. Last, focusing on cutpoint strategies does not seem too restrictive, given that the behavior of individuals is highly consistent with this type of play, as previously documented.

In the logit parameterization of the cutpoint QRE, the distribution over cutpoint strategies used by player 2 has the standard property. Namely, the log odds of player 1 choosing any cutpoint $c$ versus any other cutpoint $c^{\prime}$ is proportional to the ex ante difference in expected payoffs between using those two cutpoints, and similarly for player 2 .

Logit QRE in Behavioral Strategies.-For any response parameter $\lambda$, we solve for a fixed point in behavioral strategies. Denote by $\phi_{\lambda}^{*}$ such an equilibrium fixed point and by $\phi_{\lambda}^{*}(s)$ the equilibrium probability of fighting given a strength $s$.

First, consider the simultaneous game. We need to determine the expected utility of $\phi$ for a player with strength $s$ conditional on the other player using strategy $\phi_{\lambda}^{*}$ and having chosen $\rho$. This is simply equal to the conditional probability that the other player has strength less than $s$, given that he has chosen $\rho$. It is then given by

$$
V_{\phi}\left(s ; \phi_{\lambda}^{*}\right)=\frac{\int_{0}^{s}\left[1-\phi_{\lambda}^{*}(t)\right] d t}{\int_{0}^{1}\left[1-\phi_{\lambda}^{*}(t)\right] d t} .
$$

The expected utility of $\rho$ conditional on the other player having chosen $\rho$ is simply $V_{\rho}\left(s ; \phi_{\lambda}^{*}\right)=M$, so the difference in the expected utility of $\phi$ and $\rho$ is

$\Delta\left(s ; \phi_{\lambda}^{*}\right)=\int_{0}^{1}\left[1-\phi_{\lambda}^{*}(t)\right] d t\left(V_{\phi}\left(s ; \phi_{\lambda}^{*}\right)-V_{\rho}\left(s ; \phi_{\lambda}^{*}\right)\right)=\int_{0}^{s}\left[1-\phi_{\lambda}^{*}(t)\right] d t-M \int_{0}^{1}\left[1-\phi_{\lambda}^{*}(t)\right] d t$.

Hence, in a symmetric logit QRE, $\phi_{\lambda}^{*}$ is characterized by

$$
\phi_{\lambda}^{*}(s)=\frac{e^{\lambda \Delta\left(s ; \phi_{\lambda}^{*}\right)}}{1+e^{\lambda \Delta\left(s ; \phi_{\lambda}^{*}\right)}} \quad \text { for all } s \in[0,1] .
$$

The sequential game requires solving simultaneously for $\phi_{\lambda 1}^{*}\left(s_{1}\right)$ and $\phi_{\lambda 2}^{*}\left(s_{2}\right)$. The expressions for the first mover are exactly the same as in the simultaneous move game. Therefore, modifying the notation slightly to make clear that it is player 1's equation, we get

$$
\phi_{\lambda 1}^{*}\left(s_{1}\right)=\frac{e^{\lambda \Delta_{1}\left(s_{1} ; \phi_{\lambda 2}^{*}\right)}}{1+e^{\lambda \Delta_{1}\left(s_{1} ; \phi_{\lambda 2}^{*}\right)}} \quad \text { for all } s_{1} \in[0,1]
$$


where

$$
\Delta_{1}\left(s_{1} ; \phi_{\lambda 2}^{*}\right)=\int_{0}^{s_{1}}\left[1-\phi_{\lambda 2}^{*}(t)\right] d t-M \int_{0}^{1}\left[1-\phi_{\lambda 2}^{*}(t)\right] d t
$$

The condition for the second mover is the same, except the second mover's expected utility difference does not have to be conditioned on the first mover choosing $\rho$. We get

$$
\phi_{\lambda 2}^{*}\left(s_{2}\right)=\frac{e^{\lambda \Delta_{2}\left(s_{2} ; \phi_{\lambda 1}^{*}\right)}}{1+e^{\lambda \Delta_{2}\left(s_{2} ; \phi_{\lambda 1}^{*}\right)}} \quad \text { for all } s_{2} \in[0,1]
$$

where

$$
\Delta_{2}\left(s_{2} ; \phi_{\lambda 1}^{*}\right)=\frac{\int_{0}^{s_{2}}\left[1-\phi_{\lambda 1}^{*}(t)\right] d t}{\int_{0}^{1}\left[1-\phi_{\lambda 1}^{*}(t)\right] d t}-M .
$$

Logit QRE in Cutpoint Strategies.-Next, we consider the slightly more sophisticated version of QRE, where players are assumed to randomize over monotone cutpoint strategies, which we call QRE-cut. In our game, a cutpoint strategy is a critical value of strength, $c$, such that player $i$ chooses $\phi$ if $s_{i} \geq c$ and chooses $\rho$ if $s_{i}<c$. Hence, we define a cutpoint quantal response to be given by two probability distributions over $c$, one for each player, denoted $q_{1}(c)$ and $q_{2}(c)$. In the simultaneous version of the game, we consider only symmetric QRE-cut, where $q_{1}(c)=q_{2}(c)$ $=q(c)$ for all $c$. For the sequential version, generally $q_{1}(c) \neq q_{2}(c)$, since it is not a symmetric game and the second player chooses a cutpoint after observing the first player's move. We use the logit quantal response function for a parametric specification. Hence, the probability that a player chooses a particular strategy is proportional to the exponentiated expected payoff from using that strategy, given the cutpoint quantal response function of the other player. It is worth noting that past studies have found that in binary choice games with continuous types, a cutpoint strategy can be a useful variation on the standard QRE approach (Casella, Gelman, and Palfrey 2006). Furthermore, the analysis in Section IIIC suggests that subjects adhere to this type of strategy.

Consider the simultaneous game. The expected utility to player 1 of using a cutpoint strategy $\tilde{c}$ if player 2 uses $q(\cdot)$ is given by

$$
U(\tilde{c})=\int_{\tilde{c}}^{1} s d s+\int_{0}^{\tilde{c}}\left[\int_{0}^{s} q(c)(c M+(s-c)) d c+\int_{s}^{1} q(c) c M d c\right] d s .
$$

The first term is the probability of drawing a strength, $s$, above the cutpoint in which case player 1 chooses $\phi$ and obtains a payoff one only if player 2 has a lower strength. The second term is the probability of drawing a strength, $s$, below the cutpoint in which case player 1 chooses $\rho$. Then, if player 2's strength is lower, a compromise gives payoff $M$ and a no compromise gives payoff one. If player 2's strength 
is higher, a compromise gives payoff $M$, and a no compromise gives payoff zero. In a symmetric logit QRE-cut,

$$
q(\tilde{c})=\frac{e^{\lambda U(\tilde{c})}}{\int_{0}^{1} e^{\lambda U(c)} d c} \text { for all } \tilde{c} \in[0,1] .
$$

In the sequential game, the expression for the first mover's utility of using $\tilde{c}$, given player 2 uses $q_{2}(\cdot)$ is the same as in the simultaneous case:

$$
U_{1}(\tilde{c})=\int_{\tilde{c}}^{1} s_{1} d s_{1}+\int_{0}^{\tilde{c}}\left[\int_{0}^{s_{1}} q_{2}(c)\left(c M+\left(s_{1}-c\right)\right) d c+\int_{s_{1}}^{1} q_{2}(c) c M d c\right] d s_{1} .
$$

By contrast, the second mover's utility of using $\tilde{c}$, given player 1 uses $q_{1}(\cdot)$ does not have to be conditioned on the first mover choosing $\rho$. That is,

$$
U_{2}(\tilde{c})=\int_{\tilde{c}}^{1}\left[\frac{\int_{0}^{s_{2}} c_{1} q_{1}\left(c_{1}\right) d c_{1}}{\int_{0}^{1} c_{1} q_{1}\left(c_{1}\right) d c_{1}}+\frac{\int_{s_{2}}^{1} s_{2} q_{1}\left(c_{1}\right) d c_{1}}{\int_{0}^{1} c_{1} q_{1}\left(c_{1}\right) d c_{1}}\right] d s_{2}+\tilde{c} M .
$$

There are three observations to make about the QRE-cut solutions. First, in the sequential game, the equilibrium cutpoint distributions are different for the two players. The second mover generally adopts lower cutpoints, which translates into higher $\phi$ rates. Second, players adopt lower cutpoints when $M$ is lower. Third, the cutpoint distributions for the first mover in the sequential games are different from the cutpoint distributions in the corresponding simultaneous games, even though the utility formulas (equations 2 and 3) are identical.

We fit the behavioral strategy logit QRE and the cutpoint strategy logit QRE models by standard maximum likelihood techniques, i.e., finding the value of $\lambda$ that maximizes the likelihood of the observed frequencies of strategies. We estimate restricted and unrestricted versions of the models. In the most restricted version, the parameters are constrained to be the same across all treatments. We also estimate a version where the parameters are constrained to be the same for the 0.39 and 0.50 treatments, but are allowed to be different in the simultaneous and sequential games.

\section{B. Cognitive Hierarchy}

The cognitive hierarchy model (Camerer, Ho, and Chong 2004) postulates that when a player makes a choice, his decision process corresponds to a "level of sophistication," $k$, with probability $p_{k}$. The $\mathrm{CH}$ solution to a game is uniquely determined by an assumption about how level 0 types behave $\left(\sigma_{0}\right)$, and the distribution of levels of sophistication 11 Once the behavior of level 0 players is determined, level 1

\footnotetext{
${ }^{11}$ The CH model is an extension of the original level- $k$ model of Nagel (1995). See Dale O. Stahl and Paul W. Wilson (1995), Vincent P. Crawford and Nagore Iriberri (2007), Miguel A. Costa-Gomes and Crawford (2006),
} 
players are characterized by choosing with equal probability all strategies that are best responses to level 0 opponents. Level 2 players optimize assuming they face a distribution of level 0 and level 1 players, where the distribution satisfies truncated rational expectations. That is, the beliefs of level 2 players that their opponent is choosing according to a level 0 or a level 1 decision process, denoted $b^{2}(0)$ and $b^{2}(1)$, is given by the truncated "true" distribution of these types, $b^{2}(0)=p_{0} /\left(p_{0}+p_{1}\right)$ and $b^{2}(1)=p_{1} /\left(p_{0}+p_{1}\right)$. Level 2 players are then characterized by choosing with equal probability all strategies that are best responses to $b^{2}$ beliefs about the opponents. Higher levels are defined analogously, so a level $k$ optimizes with respect to beliefs $b^{k}$, where $b^{k}(j)=p_{j} / \sum_{l=0}^{k-1} p_{l}$ for all $j \in\{1, \ldots, k-1\}$.

For any distribution of levels, $p$, this implies a unique specification of a mixed strategy for each level, $\sigma(p)=\left(\sigma_{0}(p), \ldots, \sigma_{k}(p), \ldots\right)$, and this specification can be solved recursively, starting with the lowest types. This generates predictions about the aggregate distribution of actions, denoted $\bar{\sigma}(p)=\sum_{k=0}^{\infty} p_{k} \sigma_{k}(p)$. In all applications to date, $p$ is assumed to be Poisson distributed with mean $\tau$, that is, $p_{k}=\tau^{k} e^{-\tau} /(k !)$ for all $k$. We consider two specifications of the behavior of level 0 types.

Random Actions. - In the standard $\mathrm{CH}$ model, level 0 players are typically assumed to choose an action randomly. In the context of our game, this means that they are equally likely to select $\phi$ or $\rho$, independent of their strength. Level 1 types best respond to level 0 types. It can be shown easily that the best response strategy is to choose cutpoint $M$. Level 2 players then optimize with a cutpoint somewhere between $M$ (the best response if everyone is level 0 ) and $M^{2}$ (the best response if everyone is level 1), with the exact value depending on $p_{0}$ and $p_{1}$. Behavior by higher level players is defined recursively.

Random Cutpoints.-An alternative version, which we call the cutpoint cognitive hierarchy model or $\mathrm{CH}$-cut, replaces the assumption that level 0 types randomize uniformly over actions, with the assumption that they randomize uniformly over cutpoint strategies. This implicitly endows level 0 types with some amount of rationality in the form of monotone behavior. They are more likely to choose $\phi$ when their strength is high than when their strength is low. In our game, a level 0 type who randomizes over cutpoints has a probability of fighting as a function of $s$, which is equal to $s$. As in the standard $\mathrm{CH}$, the best responses of higher types will be unique cutpoints, and are easily calculated by recursion. Since a level 0 type has a probability $1-s$ of choosing $\rho$, the posterior distribution of strength of a level 0 type conditional on choosing $\rho$ is

$$
f(s \mid \rho)=\frac{1-s}{\int_{0}^{1}(1-x) d x}=2-2 s
$$

and Camerer, Palfrey, and Rogers (2006) for further examples of estimation of level- $k$ models based on experimental data. 
Hence, the expected payoff of $\phi$ for a level 1 type with strength $s$, and conditional on the other player being level 0 and choosing $\rho$, is $\int_{0}^{s}(2-2 x) d x=2 s-s^{2}$. Since the payoff of $\rho$ is $M$, the optimal cutpoint of a level 1 type is the value $s_{1}^{M}$ that solves $2 s_{1}^{M}-\left(s_{1}^{M}\right)^{2}=M$, that is $s_{1}^{M}=1-\sqrt{1-M}$. For our two treatments, we get $s_{1}^{0.50}$ $=1-\sqrt{1 / 2} \approx 0.29$ and $s_{1}^{0.39}=1-\sqrt{11 / 18} \approx 0.22$. Higher types are then defined recursively, with the exact cutpoint for a level $k$ depending on $\left\{p_{l}\right\}_{l=0}^{k-1}$. This produces a $\mathrm{CH}$ model that is comparable to QRE in the sense that all players choose cutpoint strategies, so $\phi$ probabilities are monotone in $s$ for all players.

We fit the Poisson specification of the $\mathrm{CH}$ and cutpoint $\mathrm{CH}$ models to the dataset by finding the value of $\tau$ that maximizes the likelihood of the observed aggregate frequencies of strategies, under the assumption that types are identically and independently distributed draws. We estimate the best-fitting values of $\tau$ by maximum the likelihood for each of the four treatments, and report both constrained and unconstrained estimates.

\section{Combining Quantal Response and Strategic Hierarchies (TQRE)}

The predictions of the $\mathrm{CH}$ and $\mathrm{CH}$-cut models differ from the QRE and QRE-cut models in two important ways. First, in $\mathrm{CH}$ models, all players with the same level of sophistication choose the same cutpoint strategy. Second, predictions in $\mathrm{CH}$ are identical for the sequential and simultaneous versions of the game. Neither "bunching" by layers of reasoning nor identical behavior in the simultaneous and sequential treatments are observed in the data.

An approach that combines quantal response and hierarchical thinking, called Truncated Quantal Response Equilibrium (TQRE), is developed in Camerer, Palfrey, and Rogers (2006). This model introduces a countable number of players' skill lev$e l s, \lambda_{0}, \lambda_{1}, \ldots, \lambda_{k}, \ldots$. The distribution of skill levels in the population is given by $p_{0}, p_{1}, \ldots, p_{k}, \ldots$. A player with skill level $k$ chooses stochastically with a logit quantal response function with precision $\lambda_{k}$. TQRE assumes truncated, rational expectations in a similar manner to $\mathrm{CH}$. A player with precision $\lambda_{k}$ has beliefs $p_{j}^{k}=p_{j} / \sum_{l=0}^{k-1} p_{l}$ for $j<k$ and $p_{j}^{k}=0$ for $j \geq k$. For reasons of parsimony and comparability to $\mathrm{CH}$, we assume that skill levels are Poisson distributed and equally spaced $\lambda_{k}=\gamma k$. Thus, it is a two parameter model with Poisson parameter, $\tau$, and a spacing parameter, $\gamma$.

The TQRE model has two effects. It smooths out the mass points, and it makes different predictions for the sequential and simultaneous games. These effects work slightly differently with behavioral strategies and with cutpoint strategies, so we estimate both versions.

\section{Cursed Equilibrium}

In a CE model, players are assumed to systematically underestimate the correlation between the opponents' action and information. As in the $\mathrm{CH}$ model, a cursed equilibrium will be the same in both the sequential and simultaneous treatments. In an $\alpha$-cursed equilibrium $\left(\mathrm{CE}_{\alpha}\right)$ all players are $\alpha$-cursed. However, players believe that opponents are $\alpha$-cursed with probability $(1-\alpha)$, and they believe that actions of opponents are independent of their information with probability $\alpha$. All players 
optimize with respect to this (incorrect) mutually held belief about the joint distribution of opponents' actions and information. In our model, we can easily compute the cutpoint strategy in $\mathrm{CE}_{\alpha}$ as a function of $M$, denoted $s_{\alpha}^{*}(M)$. For a player with strength $s_{i}$, and assuming the other player is using $s_{\alpha}^{*}(M)$, the expected utility of $\phi$, conditional on the opponent choosing $\rho$ is given by

$$
\begin{aligned}
V_{\phi}^{\alpha}\left(s_{i}\right) & =\alpha \operatorname{Pr}\left\{s_{j}<s_{i}\right\}+(1-\alpha) \operatorname{Pr}\left\{s_{j}<s_{i} \mid a_{j}=\rho, s_{\alpha}^{*}(M)\right\} \\
& =\alpha s_{i}+(1-\alpha) \min \left\{1, \frac{s_{i}}{s_{\alpha}^{*}(M)}\right\} .
\end{aligned}
$$

A player with strength equal to the equilibrium cutpoint must be indifferent between $\phi$ and $\rho$. Formally, $V_{\phi}^{\alpha}\left(s_{\alpha}^{*}(M)\right)=V_{\rho}^{\alpha}\left(s_{\alpha}^{*}(M)\right)$. Therefore, 12

$$
s_{\alpha}^{*}(M)=\left\{\begin{array}{ll}
1-\frac{1-M}{\alpha} & \text { if } \alpha>1-M \\
0 & \text { if } \alpha \leq 1-M
\end{array} .\right.
$$

A difficulty with $\mathrm{CE}_{\alpha}$ is that it cannot be fit to the data due to a zero-likelihood problem: for each $\alpha$ it makes a point prediction. Therefore, we slightly modify the equilibrium concept in order to allow for stochastic choice. The approach we follow is to combine QRE with $\mathrm{CE}_{\alpha} \cdot 13$ In the simultaneous move game, a (symmetric) $\alpha$-QRE is a behavior strategy, or a set of probabilities of choosing $\phi$, one for each value of $s \in[0,1]$. We denote such a strategy evaluated at a specific strength value by $\phi(s)$. Given $\lambda$ and $\alpha$, we denote by $\alpha$-QRE the behavior strategy $\phi_{\lambda \alpha}^{*}$. If player $j$ is using $\phi_{\lambda \alpha}^{*}$ and player $i$ is $\alpha$-cursed, then $i$ 's expected payoff from choosing $\phi$ when $s_{i}=s$ is given by

$$
\begin{aligned}
V_{\phi}^{\alpha}(s)= & \int_{0}^{1} \phi_{\lambda \alpha}^{*}(t) d t\left[\alpha s+(1-\alpha) \operatorname{Pr}\left\{s_{j}<s \mid a_{j}=\phi, \phi_{\lambda \alpha}^{*}\right\}\right] \\
& +\int_{0}^{1}\left[1-\phi_{\lambda \alpha}^{*}(t)\right] d t\left[\alpha s+(1-\alpha) \operatorname{Pr}\left\{s_{j}<s \mid a_{j}=\rho, \phi_{\lambda \alpha}^{*}\right\}\right] \\
= & \alpha s \int_{0}^{1} \phi_{\lambda \alpha}^{*}(t) d t+(1-\alpha) \int_{0}^{s} \phi_{\lambda \alpha}^{*}(t) d t \\
& +\alpha s \int_{0}^{1}\left[1-\phi_{\lambda \alpha}^{*}(t)\right] d t+(1-\alpha) \int_{0}^{s}\left[1-\phi_{\lambda \alpha}^{*}(t)\right] d t .
\end{aligned}
$$

\footnotetext{
${ }^{12}$ In a fully cursed equilibrium $(\alpha=1)$, all players choose strategies as if there is no correlation between the opponent's action and information. Thus, they all behave like a level 1 player in $\mathrm{CH}$ with random actions, $s_{1}^{*}(M)$ $=M$.

${ }^{13}$ Note that player heterogeneity with respect to $\alpha$ would not solve the zero-likelihood problem. For any cursedness $\alpha \in[0,1]$, it is always true that $s_{\alpha}^{*}(M) \leq M$. However, in our data set, we have many observations where players with strength $s>M$ choose $\rho$.
} 
And the expected payoff from choosing $\rho$ is

$$
V_{\rho}^{\alpha}(s)=\alpha s \int_{0}^{1} \phi_{\lambda \alpha}^{*}(t) d t+(1-\alpha) \int_{0}^{s} \phi_{\lambda \alpha}^{*}(t) d t+M \int_{0}^{1}\left[1-\phi_{\lambda \alpha}^{*}(t)\right] d t .
$$

Using the logit specification for the quantal response function, we then apply logit choice probabilities to the difference in the expected payoff from $\phi$ and $\rho$ for each $s_{i}$ $=s$. By inspection of $V_{\phi}^{\alpha}(s)$ and $V_{\rho}^{\alpha}(s)$, this difference is

$$
\Delta\left(s ; \phi_{\lambda \alpha}^{*}\right)=\alpha s \int_{0}^{1}\left[1-\phi_{\lambda \alpha}^{*}(t)\right] d t+(1-\alpha) \int_{0}^{s}\left[1-\phi_{\lambda \alpha}^{*}(t)\right] d t-M \int_{0}^{1}\left[1-\phi_{\lambda \alpha}^{*}(t)\right] d t
$$

and the $\alpha$-QRE in the simultaneous game is then characterized by

$$
\phi_{\lambda \alpha}^{*}(s)=\frac{e^{\lambda \Delta\left(s ; \phi_{\lambda \alpha}^{*}\right)}}{1+e^{\lambda \Delta\left(s ; \phi_{\lambda \alpha}^{*}\right)}} \quad \text { for all } s \in[0,1]
$$

which can be solved numerically, for any value of $\alpha$.

In the sequential version of the game, we simultaneously need to solve for the first and second movers, $\phi_{\lambda \alpha 1}^{*}$ and $\phi_{\lambda \alpha 2}^{*}$, respectively. The expected payoff equations under $\phi$ and $\rho$ for the first mover are the same as in the simultaneous move game. So, we have

$$
\begin{aligned}
V_{\phi 1}^{\alpha}\left(s_{1}\right)= & \alpha s_{1} \int_{0}^{1} \phi_{\lambda \alpha 2}^{*}\left(s_{2}\right) d s_{2}+(1-\alpha) \int_{0}^{s_{1}} \phi_{\lambda \alpha 2}^{*}\left(s_{2}\right) d s_{2} \\
& +\alpha s_{1} \int_{0}^{1}\left[1-\phi_{\lambda \alpha 2}^{*}\left(s_{2}\right)\right] d s_{2}+(1-\alpha) \int_{0}^{s_{1}}\left[1-\phi_{\lambda \alpha 2}^{*}\left(s_{2}\right)\right] d s_{2} \\
V_{\rho 1}^{\alpha}\left(s_{1}\right)= & \alpha s_{1} \int_{0}^{1} \phi_{\lambda \alpha 2}^{*}\left(s_{2}\right) d s_{2}+(1-\alpha) \int_{0}^{s_{1}} \phi_{\lambda \alpha 2}^{*}\left(s_{2}\right) d s_{2}+M \int_{0}^{1}\left[1-\phi_{\lambda \alpha 2}^{*}\left(s_{2}\right)\right] d s_{2} .
\end{aligned}
$$

However, the expressions for the second mover are different, because expected payoffs are conditional on the observation that the first mover chose $\rho$ :

$$
\begin{aligned}
& V_{\phi 2}^{\alpha}\left(s_{2}\right)=\alpha s_{2}+(1-\alpha) \frac{\int_{0}^{s_{2}}\left[1-\phi_{\lambda \alpha 1}^{*}\left(s_{1}\right)\right] d s_{1}}{\int_{0}^{1}\left[1-\phi_{\lambda \alpha 1}^{*}\left(s_{1}\right)\right] d s_{1}} ; \\
& V_{\rho 2}^{\alpha}\left(s_{2}\right)=M .
\end{aligned}
$$


So, the payoff differences for the first and second movers are, respectively:

$$
\begin{aligned}
& \Delta_{1}\left(s_{1} ; \phi_{\lambda \alpha 2}^{*}\right)=\int_{0}^{1}\left[1-\phi_{\lambda \alpha 2}^{*}\left(s_{2}\right)\right] d s_{2}\left[\alpha s_{1}+(1-\alpha) \frac{\int_{0}^{s_{1}}\left[1-\phi_{\lambda \alpha 2}^{*}\left(s_{2}\right)\right] d s_{2}}{\int_{0}^{1}\left[1-\phi_{\lambda \alpha 2}^{*}\left(s_{2}\right)\right] d s_{2}}-M\right] \\
& \Delta_{2}\left(s_{2} ; \phi_{\lambda \alpha 1}^{*}\right)=\alpha s_{2}+(1-\alpha) \frac{\int_{0}^{s_{2}}\left[1-\phi_{\lambda \alpha 1}^{*}\left(s_{1}\right)\right] d s_{1}}{\int_{0}^{1}\left[1-\phi_{\lambda \alpha 1}^{*}\left(s_{1}\right)\right] d s_{1}}-M .
\end{aligned}
$$

Note that the RHS of $\Delta_{2}$ is similar to the RHS of $\Delta_{1}$, except for the factor of $\int_{0}^{1}\left[1-\phi_{\lambda \alpha 2}^{*}\left(s_{2}\right)\right] d s_{2}$. Since this factor is smaller than one, it means that the payoff differences to player 2 are magnified relative to player 1 , which, in equilibrium, will result in $\phi_{\lambda \alpha 2}^{*}$ having higher slope and lower mean compared to $\phi_{\lambda \alpha 1}^{*}$. The two logit equilibrium conditions are:

$$
\begin{array}{ll}
\phi_{\lambda \alpha 1}^{*}\left(s_{1}\right)=\frac{e^{\lambda \Delta_{1}\left(s_{1} ; \phi_{\lambda \alpha 2}^{*}\right)}}{1+e^{\lambda \Delta_{1}\left(s_{1} ; \phi_{\lambda \alpha 2}^{*}\right)}} \quad \text { for all } s_{1} \in[0,1], \\
\phi_{\lambda \alpha 2}^{*}\left(s_{2}\right)=\frac{e^{\lambda \Delta_{2}\left(s_{2} ; \phi_{\lambda \alpha 1}^{*}\right)}}{1+e^{\lambda \Delta_{2}\left(s_{2} ; \phi_{\lambda \alpha 1}^{*}\right)}} \quad \text { for all } s_{2} \in[0,1] .
\end{array}
$$

One can fit the logit version of the $\alpha$-QRE model to the dataset by finding the values of $\lambda$ and $\alpha$ that maximize that likelihood of the observed frequencies of strategies. As for the previous models, we report both constrained and unconstrained estimates.

\section{E. Models of Pro-social Behavior}

We also considered an alternative class of models which are not based on cognitive limitation but, instead, are founded on social preferences. There are a number of candidates from this growing family of models. We consider three. One is the fairness model by Ernst Fehr and Klaus M. Schmidt (1999). In that model, the utility of individual $i$ when he gets payoff $x_{i}$ and individual $j$ gets payoff $x_{j}$ is

$$
U_{i}\left(x_{i}, x_{j}\right)=x_{i}-\alpha \max \left\{x_{j}-x_{i}, 0\right\}-\beta \max \left\{x_{i}-x_{j}, 0\right\},
$$

where $\beta \leq \alpha$ and $0 \leq \beta<1$. For our game, the model implies that the utility payoff to each agent for winning, compromising, and losing is $1-\beta, M$, and $-\alpha$, respectively. This implies that if fairness considerations are sufficiently strong $(\beta \geq 1-$ $M)$, the equilibrium unravelling goes in the opposite direction, and all agents always play $\rho$, regardless of their strength. Otherwise, agents want to set a lower cutpoint than their rival, and we are back to the Nash equilibrium prediction where all agents 
always play $\phi$. Our subjects do not exhibit such extreme "boundary" behavior, neither individually nor in the aggregate. Thus, one would need to add other parameters or assumptions in order for this model to explain the choices of our subjects.

A second model is altruism, where a player's utility is the weighted average of his own payoff and the other agent's payoff:

$$
U_{i}\left(x_{i}, x_{j}\right)=\gamma x_{i}+(1-\gamma) x_{j}
$$

This model runs into the same problem as the previous one. Each player's payoff of winning, compromising, and losing is $\gamma, M$, and $1-\gamma$, respectively. Therefore, all players should either always fight or always retreat. Given estimates of $\gamma$ from other experiments $(\gamma>0.5)$, the model predicts that players should always fight if $M \leq 0.50$.

Third, models based on reciprocity are also prominent in the social preferences literature. These models provide an explanation for the behavior commonly observed in the trust game. In our setting, they suggest that second movers should fight less than first movers, as they are "returning the favor" of compromising. However, we find the opposite: observing $\rho$ significantly decreases the willingness to reciprocate by responding also with $\rho$.

Overall, these leading models of social preferences described above-fairness, altruism, and reciprocity - fail to explain the basic patterns we observe in the data. Therefore, we do not estimate them. In fact, there are at least two additional reasons why models of prosocial behavior are unsuitable to account for the choices of subjects in this particular game. First, each individual plays the game many times (40), anonymously, against a pool of opponents, with the roles of players and the strengths randomly assigned in each match. Given this design, applying models of social preferences to behavior in isolated games is questionable a priori. In fact, in one of the designs $(M=0.50)$, subjects play a constant sum game against changing opponents. Hence, deviations from optimal best replies to "the field" will necessarily, over the course of the 40 matches, give a player a total expected payoff below the average payoff of the other subjects. Second and related to the above argument, in this game selfish play leads to ex ante fair and efficient allocations. Indeed, for the case of $M=0.39$, myopic "fair" behavior (everyone retreating every time) leads to long run inefficient outcomes and no long run improvement in the equality of payoffs. Subjects would be leaving over 20 percent of potential group earnings on the table, with virtually zero gain in equality of outcomes.

\section{F. Model Estimates}

In this section, we estimate the QRE, CH, TQRE, and CE models. We explore the stability of the estimated parameters across the different treatments, and compare the ability of these models to capture the basic features of the data identified in the previous section. We report the estimates in Table 8 at different levels of aggregation: for the treatments separately, pooling across the $M$-treatments, and pooling across all treatments. For the QRE, CH, and TQRE models, we considered both the behavioral strategy version and the cutpoint version. In all three models, the cutpoint version fits the data better than the behavioral strategy version in every 
TABLe 8-Model Estimates

\begin{tabular}{|c|c|c|c|c|c|c|c|c|c|c|c|}
\hline & \multirow[b]{2}{*}{$N$} & \multicolumn{2}{|c|}{$Q R E$} & \multicolumn{2}{|c|}{$\mathrm{CH}$} & \multicolumn{3}{|c|}{$T Q R E$} & \multicolumn{3}{|c|}{$\alpha-Q R E$} \\
\hline & & $\lambda$ & $-\ln L$ & $\tau$ & $-\ln L$ & $\gamma$ & $\tau$ & $-\ln L$ & $\lambda$ & $\alpha$ & $-\ln L$ \\
\hline Sim 0.39 & 560 & 20.8 & 171.0 & 0.6 & 183.2 & 4.4 & 5.0 & 170.5 & 26.6 & 0.92 & 145.5 \\
\hline $\operatorname{Sim} 0.50$ & 560 & 11.3 & 213.6 & 0.3 & 211.7 & 449.0 & 0.4 & 210.3 & 18.4 & 0.77 & 202.9 \\
\hline Sim All & 1,120 & 16.2 & 387.8 & 0.5 & 397.4 & 6.9 & 2.7 & 386.3 & 21.3 & 0.85 & 355.6 \\
\hline Seq 0.39 & 395 & 11.5 & 125.0 & 0.4 & 137.1 & 6.0 & 2.4 & 124.6 & 23.5 & 0.97 & 102.0 \\
\hline Seq 0.50 & 386 & 9.3 & 140.5 & 0.5 & 137.9 & 142.0 & 0.5 & 136.6 & 15.8 & 0.75 & 138.2 \\
\hline Seq All & 781 & 10.4 & 265.3 & 0.4 & 275.1 & 8.0 & 1.8 & 263.5 & 18.4 & 0.86 & 248.9 \\
\hline All & 1,901 & 13.0 & 656.8 & 0.5 & 672.6 & 10.0 & 1.8 & 651.2 & 20.1 & 0.85 & 605.9 \\
\hline
\end{tabular}

single treatment and in all the pooled estimations. This is not surprising, given our earlier finding that most subjects exhibit choice behavior that is consistent with a cutpoint strategy. We therefore report and discuss only the results for the cutpoint versions of these models.

Figure 3 displays the empirical and fitted fighting probabilities as a function of strength. Fitted choice frequencies in the figure are based on out-of-sample parameter estimates. Specifically, the displayed curves for the sequential data are constructed using the parameter estimates obtained from the pooled simultaneous data, and vice versa. All these models capture the upward sloping empirical frequency of $\phi$. All exhibit low $\phi$ rates for low strengths and high $\phi$ rates for high strengths.

There is some variation in fit across the different models. The better fitting models all converge to fighting probabilities of zero for low strengths and one for high strengths. The $\alpha$-QRE model, which generally fits the best of all these models, does not have cutpoints built into it explicitly, but boils down to a "soft" cutpoint model. The CH and QRE models fit the data similarly in terms of log likelihood, but there are some important differences in the predicted fight curves. As one can see from Figure 3, the $\mathrm{CH}$ model predicts somewhat better at strengths below 20, but QRE generally fits better than $\mathrm{CH}$ elsewhere. This can be attributed to two important differences in the models: (a) QRE predicts that second movers will have different (and sharper) response functions than first movers, which is a feature of the data not captured by $\mathrm{CH}$; and (b) QRE generates a smooth fight curve, while $\mathrm{CH}$ predicts clustering of cutpoints, leading to jumps in the fight curve corresponding to different levels of sophistication.

The TQRE model does not provide a substantial improvement over QRE or CH. In fact, the fitted $\phi$-rate for TQRE and QRE are very similar. They both share the problem of overestimating the fighting rates for subjects with low strength. The $\alpha$-QRE is the best fitting of all models, as it combines the elements of cursedness and stochastic choice. The pure cursed equilibrium predicts the steepest response of fighting probability as a function of strength. In fact, all players follow the same cutpoint strategy, which is a function of $\alpha$, the players' degree of cursedness. Adding quantal response produces a nice logit function of the fighting probability that crosses 0.50 at $s \approx 0.40$, varying slightly with $M$ and position, consistent with the data. Furthermore, quantal response also introduces a steeper $\phi$ curve for the second movers than for the first movers, which is again consistent with the data.

There are some differences in fit between the $M=0.39$ and the $M=0.50$ treatments, with most models fitting the data from the $M=0.39$ treatment better, reflecting 


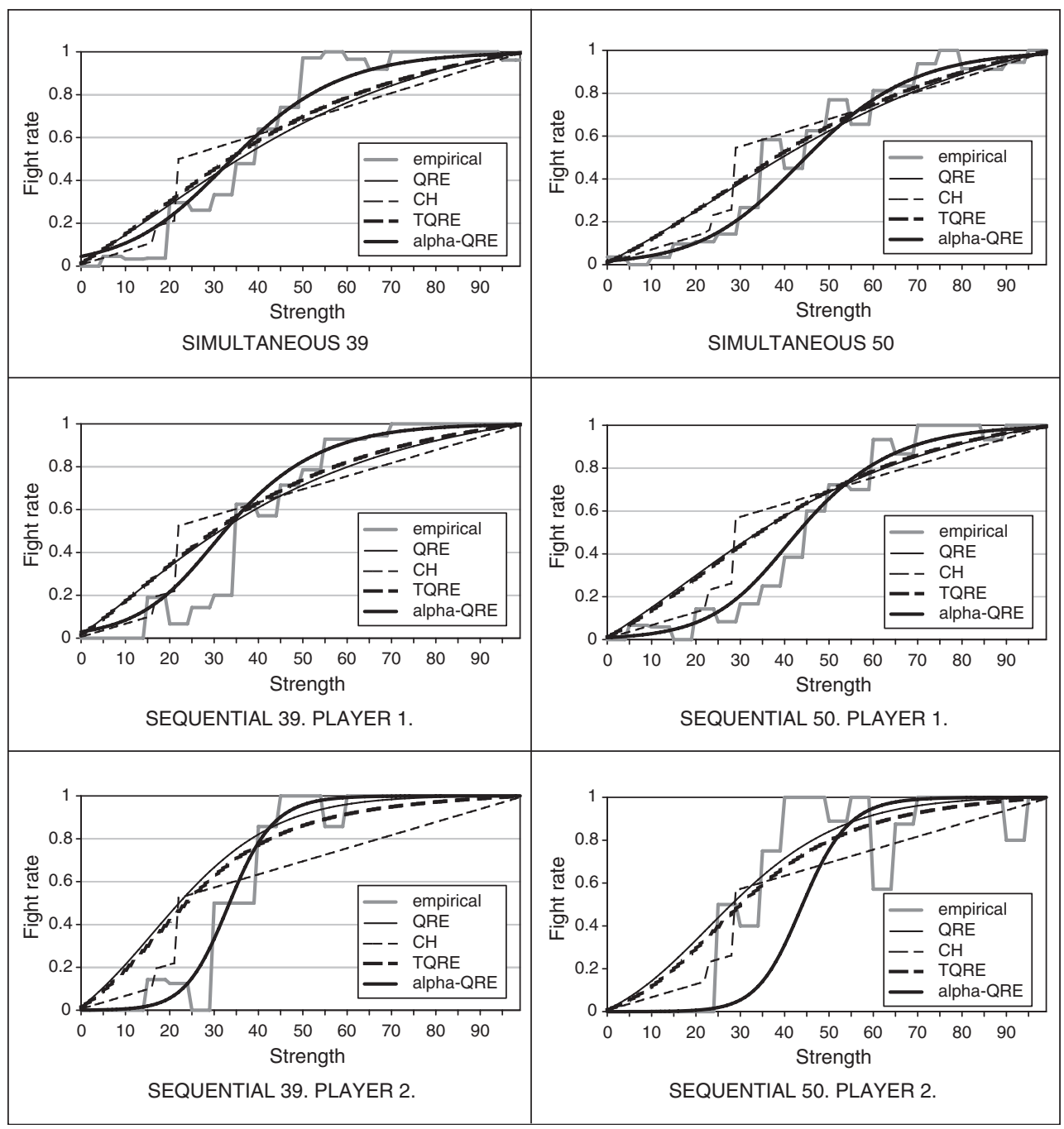

Figure 3. Empirical and Fitted Fight Rates

the steeper empirical $\phi$ curves in the $M=0.39$ data. There is virtually no difference in either the fit or the actual parameter estimates for the sequential and simultaneous treatments. The $\alpha$-QRE pooled estimates of $\lambda$ and $\alpha$ are not significantly different between the two treatments, even at the 5 percent level, and the fit is identical (log Likelihood $/ N=-0.318$ in both cases).

\section{G. Summary of Estimation Results}

The main findings about the estimated models are summarized as follows.

- All four models capture the most basic qualitative properties of behavior (none of which is consistent with Nash equilibrium). Fight rates are high but well 
below 100 percent, increasing in $s$, and decreasing in $M$. Each model captures different specific features of the data, however.

- Estimates are similar across $M$ treatments, and little power is lost by pooling treatments. This means that the results are not due to "tuning the parameters to fit the data." In fact, the out-of-sample parameters from $M=0.39$ provide virtually identical predictions for $M=0.50$ behavior as the within sample estimates.

- Only QRE and the models hybridized with QRE capture the fact that first movers behave differently from second movers. In particular, the $\phi$ function is steeper for second movers in those models.

- The cutpoint versions of $\mathrm{CH}$ and QRE describe behavior better than the behavior strategy versions. This is consistent with our findings at the individual level which indicate that over 95 percent of choices follow pure cutpoint strategies.

- TQRE provides an almost identical fit as QRE, suggesting that, in this game, the addition of hierarchical thinking to quantal response does not have a substantial impact. This is also consistent with the fact that we do not find individual cutpoints clustered around three or four strength values, as would be predicted by $\mathrm{CH}$ and other levels-of-sophistication models.

- The $\alpha$-QRE model fits the data best. The estimates of $\alpha$ are significantly greater than zero and significantly less than one. They are virtually identical for both the sequential and simultaneous games, suggesting that the two-parameter model is not overfitting the data.

\section{Conclusions}

The compromise game is obviously challenging to the cognitive abilities of players. In our experiment, players seem to understand some basic elements of the game, such as the cutpoint nature of best responses. They have problems figuring out the full logic of the unravelling argument, however.

The paper has considered several cognitive explanations for the surprising behavior observed in this game of incomplete information. In future research, it might be interesting to explore more general models. One candidate would be the "analogy-based expectation equilibrium" developed by Philippe Jehiel (2005) and Jehiel and Frederic Koessler (2006) which can be seen as an alternative version of cursed equilibrium. A second direction would be to explicitly allow for heterogeneity. While the $\mathrm{CH}$ model is suggestive of heterogeneity, the attempts here and elsewhere to fit the model assume homogeneity, since repeated observations of the same individual are treated as independent draws from the type space. In principle, one could extend the estimation of $\mathrm{CH}$ models to allow for fixed types. However, for our data, it seems unlikely to go very far because we do not observe clusters of behavior that might correspond to types-in contrast to Nagel's (1995) guessing game, for example. The QRE and $\alpha$-QRE models could also be extended to allow for heterogeneity with respect to $\lambda$ and $\alpha$, also with fixed types. This would undoubtedly lead to better fits in terms of log likelihood, but it is hard to imagine any new insights emerging from such an exercise.

One of the most interesting findings is that the order of moves affects choices. In our game, a player's action is relevant only if the rival chooses $\rho$. Thus, first, 
second, and simultaneous movers should all condition their strategy on that event. By contrast, the data shows that players who observe $\rho$ being played by their rival (second movers) respond more aggressively than players who must condition on the anticipation of that event (first movers). Even among subjects who do not observe the choice of the rival before playing, there is a difference between knowing that one's choices will be publicly observed before the rival makes his choice (first movers) and knowing that one's choices will not be observed (simultaneous movers). In sum, hypothetical conditioning on events seems to produce different behavior than observational conditioning on events. While we found one general explanation for this phenomenon (QRE), a search for other parsimonious formal models that imply different behavior between our first and second movers might add to our understanding. Such a search seems a worthy project for future research. It could also help settle questions well beyond the scope of our compromise game, such as the effect of using the strategy method in experimental games and the differences in behavior between strategically equivalent games (extensive versus strategic form). Naturally, this has implications for many strategic settings of significant applied interest, including common value auctions and voting behavior, where optimal choice requires bidders to condition on winning and voters to condition on being pivotal.

\section{REFERENCES}

-Akerlof, George A. 1970. "The Market for 'Lemons': Quality Uncertainty and the Market Mechanism.” Quarterly Journal of Economics, 84(3): 488-500.

- Camerer, Colin F., Teck-Hua Ho, and Juin-Kuan Chong. 2004. "A Cognitive Hierarchy Model of Games." Quarterly Journal of Economics, 119(3): 861-98.

Camerer, Colin F., Thomas R. Palfrey, and Brian W. Rogers. 2006. "Heterogeneous Quantal Response Equilibrium and Cognitive Hierarchies." California Institute of Technology Division of the Humanities and Social Sciences Working Paper 1260.

Casella, Alessandra, Andrew Gelman, and Thomas R. Palfrey. 2006. "An Experimental Study of Storable Votes." Games and Economic Behavior, 57(1): 123-54.

Costa-Gomes, Miguel A., and Vincent P. Crawford. 2006. "Cognition and Behavior in Two-Person Guessing Games: An Experimental Study." American Economic Review, 96(5): 1737-68.

-Crawford, Vincent P., and Nagore Iriberri. 2007. "Level-K Auctions: Can a Nonequilibrium Model of Strategic Thinking Explain the Winner's Curse and Overbidding in Private-Value Auctions?" Econometrica, 75(6): 1721-70.

Eyster, Erik, and Matthew Rabin. 2005. “Cursed Equilibrium.” Econometrica, 73(5): 1623-72.

-Fehr, Ernst, and Klaus M. Schmidt. 1999. "A Theory of Fairness, Competition, and Cooperation." Quarterly Journal of Economics, 114(3): 817-68.

Forsythe, Robert, R. Mark Isaac, and Thomas R. Palfrey. 1989. "Theories and Tests Of "Blind Bidding" In Sealed-Bid Auctions." RAND Journal of Economics, 20(2): 214-38.

Goeree, Jacob K., Charles A. Holt, and Thomas R. Palfrey. 2005. "Regular Quantal Response Equilibrium." Experimental Economics, 8(4): 347-67.

Holt, Charles A., and Roger Sherman. 1994. “The Loser's Curse.” American Economic Review, 84(3): 642-52.

-Jehiel, Philippe. 2005. "Analogy-based Expectation Equilibrium.” Journal of Economic Theory, 123(2): 81-104.

Jehiel, Philippe, and Frederic Koessler. 2006. "Revisiting Games of Incomplete Information with Analogy-Based Expectations." Games and Economic Behavior, 62(2): 533-57.

Kagel, John H., and Dan Levin. 2002. Common Value Auctions and the Winner's Curse. Princeton, NJ: Princeton University Press.

Lynch, Michael, Ross Miller, Charles Plott, and Russell Porter. 1984. "Product Quality, Consumer Information, and 'Lemons' in Experimental Markets." In Empirical Approaches to Consumer Protection Economics, ed. Pauline Ippolito and David T. Scheffman, 251-306. Washington, DC: Federal Trade Commission 
McKelvey, Richard D., and Thomas R. Palfrey. 1998. "Quantal Response Equilibria for Extensive Form Games.” Experimental Economics, 1(1): 9-41.

-McKelvey, Richard D., and Thomas R. Palfrey. 1995. "Quantal Response Equilibria for Normal Form Games." Games and Economic Behavior, 10(1): 6-38.

- Milgrom, Paul, and Nancy Stokey. 1982. "Information, Trade and Common Knowledge." Journal of Economic Theory, 26(1): 17-27.

-Morris, Stephen. 1994. "Trade with Heterogeneous Prior Beliefs and Asymmetric Information." Econometrica, 62(6): 1327-47.

-Myerson, Roger B., and Mark A. Satterthwaite. 1983. "Efficient Mechanisms for Bilateral Trading." Journal of Economic Theory, 29(2): 265-81.

Nagel, Rosemarie. 1995. "Unraveling in Guessing Games: An Experimental Study." American Economic Review, 85(5): 1313-26.

Palfrey, Thomas R., and Jeffrey E. Prisbrey. 1996. "Altruism, Reputation and Noise in Linear Public Goods Experiments." Journal of Public Economics, 61(3): 409-27.

Samuelson, William, and Max Bazerman. 1985. "The Winner's Curse in Bilateral Negotiations." In Research in Experimental Economics, Vol. 3, ed. Vernon Smith, 105-37. Greenwich, CT: JAI Press.

Sonsino, Doron, Ido Erev, and Sharon Gilat. 2001. "On Rationality, Learning and Zero-Sum Betting-An Experimental Study of the No-Betting Conjecture." Unpublished.

Sovic, Ylva. 2004. "Strength of Dominance and Depths of Reasoning." Unpublished.

-Stahl, Dale O., and Paul W. Wilson. 1995. "On Players' Models of Other Players: Theory and Experimental Evidence." Games and Economic Behavior, 10(1): 218-54.

Wang, Stephanie. 2006. "Misunderstanding the Other." Unpublished. 
This article has been cited by:

1. I. Brocas, J. D. Carrillo, S. W. Wang, C. F. Camerer. 2014. Imperfect Choice or Imperfect Attention? Understanding Strategic Thinking in Private Information Games. The Review of Economic Studies . [CrossRef]

2. Vincent P. Crawford,, Miguel A. Costa-Gomes,, Nagore Iriberri. 2013. Structural Models of Nonequilibrium Strategic Thinking: Theory, Evidence, and Applications. Journal of Economic Literature 51:1, 5-62. [Abstract] [View PDF article] [PDF with links]

3. Kyle Mattes. 2012. What Happens when a Candidate Doesn't Bark? "Cursed” Voters and Their Impact on Campaign Discourse. The Journal of Politics 1-14. [CrossRef] 